\title{
Acknowledgement to Reviewers of Diagnostics in 2019
}

\section{Diagnostics Editorial Office}

MDPI, St. Alban-Anlage 66, 4052 Basel, Switzerland

Published: 20 January 2020

The editorial team greatly appreciates the reviewers who have dedicated their considerable time and expertise to the journal's rigorous editorial process over the past 12 months, regardless of whether the papers are finally published or not. In 2019, a total of 234 papers were published in the journal, with a median time to first decision of 15.5 days and a median time from submission to publication of 34.5 days. The editors would like to express their sincere gratitude to the following reviewers for their generous contribution in 2019:

Achreja, Abhinav

Ahamed, Muneer

Ahn, Yong

Albano, Domenico

Albu, Silviu

Alizargar, Javad

Amato, Dominick J.

Amiya, Eisuke

Amza, Catalin

Anton, David

Armellini, Elia

Ashford, J. Wesson

Assenza, Giovanni

Awan, Shakil Ahmed

Babichev, Sergii

Badea, Mihaela

Bagyinszky, Eva

Bakkour, Sonia

Balbi, Bruno

Banerjee, Sudip

Banning, Antje

Barbosa, Ana Isabel

Bardin, Ron

Baroncini, Damiano

Barra, Fabio

Barzon, Luisa

Bauckneht, Matteo

Bechsgaard, Rie Eriksen
Belmin, Joël

Belteki, Gusztav

Bequette, B. Wayne

Beretta, Giovanni L.

Bhatti, Fazal-ur-Rehman

Bisharat, Naiel

Bishwajit, Ghose

Blackburn, Jessica

Blandino, Giovanni

Board, Mary

Boling, Warren W.

Bologna, Ronell

Boomer, Jonathan S.

Borghesi, Andrea

Borse, Vikrant

Bose, Tanima

Botelho, Monica C.

Boublikova, Ludmila

Boussios, Stergios

Bragazzi, Nicola Luigi

Bueno-Campaña, Mercedes

Bunaciu, Rodica $\mathrm{P}$

Burger, Michael C

Burtey, Stéphane

Caiati, Carlo

Camara, Jose Sousa

Cambridge, Geraldine

Campos Souza, Paulo Vitor 
Cañadas-De La Fuente, Guillermo A.

Candido Carvalho, Katia

Canetta, Elisabetta

Casals Terre, Jasmina

Cascini, Giuseppe Lucio

Casiraghi, Elena

Castren, Maija

Catry, Boudewijn

Cavoretto, Paolo

Cederbaum, Stephen

Chakravarty, Shatadru

Chalopin, Claire

Chang, Chia-Chen

Chang, Hsien-Chang

Chang, Ke-Vin

Chaudhari, Bimal

Chauhan, Bharesh

Chaves, Raquel

Chen, Ke-Cheng

Chen, Kuan-Fu

Chen, Yu-Chun

Cheng, Xi

Cheng, Xiang

Chien, Yin-Hsiu

Chinnappan, Mahendran

Chioncel, Ovidiu

Chladek, Grzegorz

Choi, Hojong

Choi, Sangchun

Choo, Pei Ling

Chuah, Seng-Kee

Ciaccio, Marcello

Ciudin, Andrea

Ciuoli, Cristina

Claros, Edith

Coccheri, Sergio

Colussi, Gian Luca

Comi, Cristoforo

Converse, Paul J.

Corda, Andrea

Corrochano, Silvia

Corsi, Cristiana

Costa, Pedro
Coutinho, Maria Francisca

Cox, Jonathan A.G.

Crippa, Paolo

Cristache, Corina Marilena

Cuadra, Giancarlo

Czekierdowski, Artur

Dabrowski, Filip

Daimi, Houria

Dal Ben, Matteo

Daley, Peter

Dang, Shaonong

Daniellou, Richard

Datta, Rupsa

Dayan, Michael

De Lorenzo, Mariana S

De Stefano, Rosa

Delfino, Ines

Desai, Niyati

Devarajan, Priyadharshini

Di Donato, Marzia

Di Gianfilippo, Riccardo

Dinets, Andrii V.

Dinic, Jelena

Dionysopoulos, Dimitrios

Docea, Anca Oana

Domenici, Giacomo

Dörr, Stefan

Drago, Lorenzo

Dragomir, Mihnea

Dubey, Harishchandra

Dusi, Veronica

Eboigbodin, Kevin

Ecke, Thorsten

Edmund Kim, Euishin

Ekpo, Ernest U.

El Hadi, Hamza

Elhabian, Shireen Y.

Emfietzoglou, Dimitris

Engelhardt, Paul Friedrich

Estanis, Navarro

Esteban, Emilio

Facciorusso, Antonio

Falzone, Luca 
Fang, Xiaolan

Fanti, Alessandro

Farber, Harrison W.

Farina, Nicholas H.

Fasoula, Angie

Fernández-Ochoa, Álvaro

Ferrara, Pietro

Ferraris, Claudia

Field, Michael

Filella, Xavier

Filius, Anika

Fiorillo, Luca

Fitsiori, Aikaterini

Fleischer, Arthur C.

Folkvardsen, Dorte Bek

Forest, Fabien

Forget, Patrice

Fountas, Kostas

Franco, Luis

Freitas, Leandro Oliveira

Fthenakis, George

Fujigaki, Motoharu

Fukumoto, Manabu

Fukushima, Hiroshi

Furmańczyk, Konrad

Galasso, Giovanni

Gallardo, Enrique

Gallotta, Valerio

Galvosas, Petrik

Gama, Adelina

Ganetzky, Rebecca

Gao, Shiqian Sherry

Garcia-Diaz, Julia

Garrido, Juan E.

Gasca-Salas, Carmen

Gasperini, Serena

Gerke, Oke

Germain, Arnaud

Gheorghiu, Eugen

Ghiran, Ionita C.

Giacomini, Mauro

Gijón-Nogueron, Gabriel

Giovagnoli, Raffaela
Giraldo, Pilar

Gjörloff Wingren, Anette

Gladysheva, Inna P.

Goldoni, Matteo

Gomez, Antonio

Gómez-Urquiza, Jose L.

González-López, José Rafael

González-Vallinas, Margarita

Gorte, Ben

Goyal, Rajni

Graziani, Maristella

Grewal, Raji Paul

Guerra, Flora

Gundapaneni, Dinesh

Guo, Qihao

Gupta, Vijayalaxmi

Haaparanta-Solin, Merja

Hafiane, Anouar

Hanai, Jun-ichi

Hanna, Ramy M.

Harmatz, Paul

Hartanto, Andree

Hasanian, Mostafa

Hasegawa, Takaaki

Hashemi, Parastoo

Hassan, Mohamed

Haszprunar, Gerhard

Hattori, Toshio

Hayashi, Ryuji

He, Aina

$\mathrm{He}, \mathrm{Yi}$

Hendrikse, Harry

Hernández-Torres, Francisco

Hernandez-Vaquero, Daniel

Hifumi, Toru

Hingorani, Dina

Hirata, Kenji

Hołda, Mateusz Krystian

Holt, Kelly

Holy, Richard

Horai, Yoshiro

Horn, Sebastian

Hronek, Miloslav 
$\mathrm{Hu}$, Xiaosu

Huang, Tzu-Ting

HUANG, Wen-Chin

Hubková, Beáta

Hughes, Kevin

Hwang, Jae Youn

Iacopi, Elisabetta

Ide, Kazuki

Ilich-Ernst, Jasminka

Imamura, Teruhiko

Ingendoh-Tsakmakidis, Alexandra

Inglada-Pérez, Lucía

Itri, Angelo

Jain, Kartik

Jakab, András

Janczar, Szymon

Janmaimool, Piyapong

Jašprová, Jana

Jatužis, Dalius

Jáuregui-Vázquez, Daniel

Jeong, Keun-Yeong

Jiménez-Bonilla, Julio Francisco

Jin, Li

Jing, Ran

Johnen, Georg

Jönsson, Daniel

Jørgensen, Kasper

Jozwiak, Mathieu

Juhlin, Christofer

Jung, Christian

Kairemo, Kalevi

Kalfa, Nicolas

Kang, Jeonghyun

Kant, Shiva

Kanyong, Prosper

Karlsson, Joakim

Katrinli, Seyma

Katsanos, Aristeidis H.

Kavecan, Ivana

Kawaguchiya, Mitsuyo

Kayser, Klaus

Kazufumi, Suzuki

Keeley, Jared
Kent, Christopher

Kerman, Kagan

Kerr, Jonathan

Keutzer, Joan

Khoo, Bee Luan

Khurshid, Zohaib

Kikuyama, Masataka

Kim, Jee Taek

Kim, Mee Kum

Kim, Seongho

Kim, Seongjae

Kimber-Trojnar, Żaneta

Kimura, Masahiro

Kiss, Rita

Klatt, Dieter

Knowlton, Barbara J.

Kodali, Maheedhar

Koga, Fumitaka

Koike, Takahiko

Kontsevaya, Irina

Koshiyama, Masafumi

Kotyla, Przemyslaw J.

Kozieł, Edmund

Kratochwil, Clemens

Krishna, Somashekar G.

Krupa-Kozak, Urszula

Kulkarni, Priyanka

Kumar, Ramesh

Kunz, Meik

Kuwahara, Takamichi

Lacerda, Eliana

Laganà, Antonio Simone

Lai, Wen-Fu

Laiakis, Evagelia

Landolfo, Chiara

Lang, Di

Le, Nguyen Quoc Khanh

Lederer, Martin

Lee, Changwon

Lee, Cheng-I

Lee, Chii-Ming

Lee, Huei-Jane

Lee, Jie 
Lee, Regent

Lee, Tim

Lee, Zhenghong

Leffler, Jonatan

Lehmann-Che, Jacqueline

Lepage, Cecile

Lerche, Christoph

Leung, Duncan

Leutenegger, Marcel

Li, Chenghui

Li, Chunfeng

Li, Lin

Li, Xiang-Guo

Lidbury, Brett

Lima, Ernesto

Lima, Ivan

Lin, Ching-Yu

Lin, Hsiang-Yu

Lisi, Anthony J.

Litschauer, Brigitte

Liu, Jia

Liu, Wei-Min

Liu, Yu

Llull, Laura

Loffredo, Lorenzo

Lombardi, Vincent C.

Longo, Antonio

López, Daniel

López-Pintor Muñoz, Rosa María

Losurdo, Giuseppe

Lova, Paola

Lu, Chia-Feng

Lu, Jeng-Wei

Luce-Fedrow, Alison

Luciani, Mirella

Luisetto, Roberto

Luo, Zonghua

Lupien, Andréanne

M'kacher, Radhia

Machelart, Arnaud

Macín, Stella Maris

Madala, Hanumantha Rao

Magnus, Per
Mahshid, Sara

Maia, Cláudio

Malapelle, Umberto

Mandal, Subhamoy

Mandato, Claudia

Mango, Lucio

Manis, George

Manzo, Ciro

Marateb, Hamid Reza

Marchant, David

Marcu, Loredana

Mariotti, Caterina

Markić, Joško

Marks, Haley L.

Marotte, Hubert

Marshall, Andrew G.

Martelli, Eugenio

Martinelli, Giovanni

Maruyama, Hitoshi

Masala, Carla

Masarone, Daniele

Mascitti, Marco

Massaroni, Carlo

Mastropietro, Alfonso

Matalliotakis, Michail

Matikonda, Siddharth

Matsuda, Shinji

Mauk, Michael G.

Mayer, Christopher

McCary, Lindsay M.

McEwan, Alistair

McGregor, Neil R.

Mcmorrow, Tara

McNeill, Alisdair

Mehta, Meghna

Mehta, Sunali

Melchor, Juan

Mendoza-Nuñez, Victor Manuel

Mercorelli, Paolo

Merlino, Giovanni

Meshram, Nirvedh

Metz, Stefan W.

Mihaicuta, Stefan 
Miller, Jillian Vinall

Minaga, Kosuke

Mishina, Masahiro

Mitchell, John

Mitrakas, Lampros

Moccia, Marcello

Mohamed, Salah A.

Mohamed, Sharmarke

Molenaar, Remco J.

Möller, Sören

Monni, Giovanni

Montalto, Luigi

Morbelli, Silvia

Moskot, Marta

Motloch, Lukas J

Mozos, Ioana

Musch, Mark

Mustafine, Jamila

Musteata, Mihai

Muthupillai, Raja

Naber, Kurt

Nabeta, Takeru

Nacul, Luis C

Nagaraj, Vini

Nakamura, Masato

Nakanishi, Yoshitaka

Nandi, Saikat

Narayanan, Barath

Nardini, Roberto

Nath, Shubhankar

Nedoma, Jan

Nemeș, Roxana Maria

Neogi, Ujjwal

Neri, Flavia

Neuhaus, Jochen

Neumann, Ewald

Nguyen, Binh

Nguyen, Binh P.

Nicolini, Antonello

NITTA, Naotaka

Nobre, Miguel De Araújo

Nohe, Anja

Noll-Hussong, Michael
Nonaka, Taichiro

Nouri, Aria

Novo-Veleiro, Ignacio

Oakey, Zack

Obreza, Aleš

Ochi, Shinichiro

Oftadeh, Shahin

O'Harte, Finbarr

Olesberg, Jonathon

O'Loughlin, Declan

Oosterloo, Mayke

Opdenakker, Ghislain

Orsini, Joe

Ostovar, Saeideh

Oszutowska-Mazurek, Dorota

Otomo, Takanobu

Otsuki, Takemi

Ozen, Mehmet

Padwal, Raj

Pal Choudhuri, Shreoshi

Palazón-Bru, Antonio

Palestra, Giuseppe

Palmucci, Stefano

Pampaloni, Francesco

Pan, Luying

Panzuto, Francesco

Papazafiropoulou, Athanasia

Park, Jong Kook

Park, Jun-Beom

Park, Min

Parker, Christina

Parsons, Lauren N.

Pascual, Carmen $M^{a}$ García

Patini, Romeo

Patro-Małysza, Jolanta

Pavlidis, Efstathios

Pavlik, Edward

Pavlik, Edward J.

Pavlov, Chavdar S

Pavlova, Elena

Peitsidis, Panagiotis

Pelin, Marco

Pennington, Catherine 
Pereira, Filipe

Pergament, Eugene

Pezzuto, Aldo

Piciu, Doina

Pimenoff, Ville N.

Pinter, Abraham

Piotrowska, Ewa

Piskin, Senol

Pitocco, Dario

Plaza-Díaz, Julio

Pokorn, Marko

Poku, Rosemary A.

Pop, Tudor Lucian

Potaczek, Daniel P

Pouliopoulos, Antonios

Pradotto, Luca Guglielmo

Puniya, Bhanwar Lal

Puri, Kriti

Puttreddy, Rakesh

Qian, Xuejun

Qiu, Xin

Qu, Ning

Quartu, Marina

Quinlan, Jonathan

Rahib, Lola

Rahman, Hafizur

Rahmanuddin, Syed

Rai, Vikrant

Raimondo, Diego

Rajavenkatanarayanan, Akilesh

Ramadas, Amutha

Ramaraj, Pandurangan

Ramić, Snježana

Ramirez-Gonzalez, Gustavo

Ramsingh, Davinder

Ranganathan, Shivakumar

Ratajczak, Monika

Rawla, Prashanth

Ray, Supriyo

Rehman, Michael

Reiterer, Florian

Reychler, Hervé

Reynard, Olivier
Rezania, Shahabaldin

Rizzolio, Flavio

Roberts, Drucilla J.

Rocca, Carmine

Rocca, Elena

Ročka, Saulius

Rodger, James A.

Rodríguez Hermosa, Juan Luis

Romero Morales, Carlos

Romine, William

Roque, Alicia

Rosado, Carlos

Rosenzweig, Allison

Ross, J. Kim

Roy, Dhruvajyoti

Rus, Guillermo

Russell, David G.

Saha, Progyaparamita

Sahoo, Subhransu

Saijo, Yoshifumi

Salkini, Mohamad

Sampaio, Paula

Sanchez Bornot, Jose Miguel

Santhanam, Abirami

Sardu, Celestino

Sato, Keisaku

Savopoulos, Christos

Saxer, Franziska

Scaravilli, Vittorio

Scarpa, Maurizio

Schelhaas, Sonja

Schepisi, Giuseppe

Schiltenwolf, Marcus

Schirmer, Lucas

Schmidl, Doreen

Schob, Stefan

Scholkmann, Felix

Schreiber, Michael

Scimeca, Manuel

Sergi, Consolato

Seto, Toshiyuki

Shahi, Shailesh

Shankar, Eswar 
Sharma, Isha

Sharma, Umakant

Shienfeld, Yehuda

Shimada, Yohta

Shin, Young Joo

Shinohara, Mitsuru

Shinohara, Shogo

Simakov, Srgey

Simoes, Joana

Simões, João

Simopoulou, Mara

Sinclair, Andrew

Singh, Anup

Singha, Subhankar

Sivakumar, Sushama

Sivathanu, Vivek

Skuja, Sandra

Sladkevicius, Povilas

Slart, Riemer H.J.A.

Slavik, Roger

Słomka, Artur

Smani, Younes

Sobrino, Xosé Antón Vila

Solís García Del Pozo, Julián

Solís-Lemus, José Alonso

Sonkar, Kanchan

Sorokin, Maxim I.

Sorsa, Timo

Speight, Nigel

Spínola, Hélder

Spizzo, Gilbert

Sporea, Ioan

Staderini, Edoardo

Stahelin, Robert V.

Staines, Donald

Stanley, Jone A.

Steinbichler, Teresa Bernadette

Stergiou, George $\mathrm{S}$.

Stiburkova, Blanka

Stoian, Dana

Streimikiene, Dalia

$\mathrm{Su}$, Mei-Tsz

$\mathrm{Su}$, Rui
Suciu, Bogdan Andrei

Suhail, Hamid

Sun, Meng

Sun, Zhonghua

Sung, Si-Il

Surdacki, Andrzej

Sweiss, Nadera

Symonds, Michael E.

Szubert, Sebastian

Tajiri, Naoki

Takebayashi, Shigeo

Tanaka, Naoro

Tang, Kwan Ho

Tappia, Paramjit

Tarantino, Giovanni

Tarazona-Santabalbina, Francisco José

Teng, Ru-Jeng

Tiefenboeck, Thomas M.

Todsen, Tobias

Tolstikov, Vladimir V.

Tomatsu, Shunji

Tombelli, Sara

Tomonaga, Shozo

Tong, Mingming

Toriello, Filippo

Torres, Jorge

Torres-Lagares, Daniel

Tot, Tibor

Totman, John

Tran, Anh Nhat

Tsai, Chia-Hung Dylan

Tseng, Kuang-Wen

Tsolakis, Apostolos V.

Tsuchiya, Masahiko

Tsukamoto, Tetsuo

Tuchscherr, Lorena

Tzartos, Socrates J.

Uberti, Daniela

Uhl, Eberhard

Umemura, Naoki

Unger, Elizabeth

Upadhyaya, Jasbir

Urbanelli, Lorena 
Uruha, Akinori

Usai Satta, Paolo

Usmani, Shariq

Vaculovičová, Marketa

Vafiadaki, Elizabeth

Vaibhav, Kumar

Varga, Gergely

Vashist, Sandeep

Vashist, Sandeep K.

Veilleux, Louis-Nicolas

Velikova, Tsvetelina

Venkataraman, Rajesh

Venturella, Roberta

Verghese, Shilpi

Videtta, Walter

Vidic, Jasmina

Villa, Chiara

Vinall, Ruth

Vitale, Salvatore Giovanni

Waldhör, Thomas

Wang, Dong

Wang, Fupeng

Wang, Gang

Wang, Shuangyi

Wang, Tao

Wang, Tsung-Jen

Wang, William

Wang, Yumeng

Wegrzyn, Grzegorz

Wei, Qingshan

Wilczek, Piotr

Willemse, Eline

Wlaź, Piotr

$\mathrm{Wu}$, Sheng-Nan

$\mathrm{Wu}$, Shuicai

$\mathrm{Wu}$, Wei-Ting

$\mathrm{Wu}, \mathrm{Yu}$-Jen
Wynants, Laure

Xia, Junfeng

Xin, Yin

Yamauchi, Akira

Yan, Shengmin

Yang, Chul-Su

Yang, Guang

Yang, Kun

Yaqub, Maqsood

Yeh, Hong-Zen

Yeh, Sheng Lih

Yong, Kar Wey

Yoo, Tag Keun

Yoshioka, Ken-ichi

Youssof Zadeh, Vahab

Yu, Yan Ping

Yu, Yang

Yves, Combarnous

Zafar, Muhammad Sohail

Zahid, Maliha

Zemouri, Ryad

Zhang, Chi

Zhang, Daniel Xin

Zhang, Haichong K.

Zhang, Huan

Zhang, Jinnan

Zhang, Yudong

Zhang, Zhao

Zhao, Wei

Zhao, Yang

Zheng, Minzhang

Zhou, Zhuhuang

Ziegler, Magnus

Ziolkowski, Robert

Zou, Liang

Zou, Wei

(C) 2020 by the author. Licensee MDPI, Basel, Switzerland. This article is an open access article distributed under the terms and conditions of the Creative Commons Attribution (CC BY) license (http://creativecommons.org/licenses/by/4.0/). 